\title{
Challenges and design choices for global weather and climate models based on machine learning
}

\author{
Peter D. Dueben and Peter Bauer \\ European Centre for Medium-range Weather Forecasts, Shinfield Rd, Reading, RG2 9AX, UK
}

Correspondence: Peter D. Dueben (peter.dueben@ecmwf.int)

Received: 16 June 2018 - Discussion started: 28 June 2018

Revised: 30 August 2018 - Accepted: 12 September 2018 - Published: 1 October 2018

\begin{abstract}
Can models that are based on deep learning and trained on atmospheric data compete with weather and climate models that are based on physical principles and the basic equations of motion? This question has been asked often recently due to the boom in deep-learning techniques. The question is valid given the huge amount of data that are available, the computational efficiency of deep-learning techniques and the limitations of today's weather and climate models in particular with respect to resolution and complexity.

In this paper, the question will be discussed in the context of global weather forecasts. A toy model for global weather predictions will be presented and used to identify challenges and fundamental design choices for a forecast system based on neural networks.
\end{abstract}

\section{Introduction}

In recent years, artificial intelligence and machine learning have become very important for hardware development in high-performance computing (HPC) and have attracted a large amount of public interest. Neural networks (NNs) are tools from machine learning that are used successfully within many applications such as computer vision, speech recognition and data filtering. If a sufficient amount of data are available, NNs can be trained to describe the evolution of non-linear processes. Due to the fundamentally applicationunaware character, no complete understanding of the underlying process is necessary. Very complex NNs can be trained that use more than a billion trainable parameters and millions of datasets for training on HPC architecture; see, for example, Le (2013).
On the other hand, numerical weather forecasts are computationally expensive and forecast quality reduces significantly already after a couple of days even in the best models available. Most processes in the Earth system are described by non-linear differential equations with non-linear interactions between Earth system components. Due to the complexity and size of the Earth system and the limited capacity of today's supercomputers, it is necessary to make approximations when weather prediction models are formulated and resolution is truncated in space and time. The use of limited resolution makes it necessary to parameterise processes that are not resolved explicitly within model simulations. To optimise parameterisation schemes a large number of parameters has to be tuned towards optimal model performance, and the traceability of physical laws of the underlying process as well as the physical interpretation for each parameter is often lost during this exercise. Furthermore, to perform weather predictions, a huge amount of data need to be processed and assimilated to create initial conditions. This is a process that will again cause significant errors and uncertainties. Only a rather small fraction of all observations can be assimilated into state-of-the-art weather prediction models due to the large computational cost and simplified assumptions required such as vanishing error correlation.

NNs have been used to post-process data from weather forecast models to optimise predictions; see, for example, Krasnopolsky and Lin (2012) or Rasp and Lerch (2018). NNs have also been used for radiation parameterisation in operational forecasts at ECMWF in the past (Chevallier et al., 1998, 2000; Krasnopolsky et al., 2005) as well as for the parameterisation of ocean physics (Krasnopolsky et al., 2002; Tolman et al., 2005) and convection (Krasnopolsky et al., 2013). Recently, the representation of atmospheric sub-grid 
processes using techniques from machine learning was investigated in more detail, with promising results using both NNs (Brenowitz and Bretherton, 2018; Gentine et al., 2018; Rasp et al., 2018) and random forest decision trees (O'Gorman and Dwyer, 2018). For parameterisation, NNs can be trained on observations or high-resolution model data, for example from high-resolution simulations of the same model, simulations that use super-parameterisation or large-eddy simulations, with the ambition to provide better results compared to conventional parameterisation schemes (see, for example, Schneider et al., 2018). NN parameterisation schemes can also be trained with input-output pairs of existing parameterisation schemes to emulate the behaviour and eventually replace the parameterisation scheme within forecasts. The latter is useful since NN parameterisation schemes that are based on very efficient HPC libraries such as TensorFlow (TensorFlow, 2018) for which co-designed hardware exists will in general be much more efficient compared to conventional parameterisation schemes that have a very large code base that is difficult to optimise. Speed-up factors of up to $10^{5}$ have been observed; see Krasnopolsky and Fox-Rabinovitz (2006). It is possible that NNs may become a standard tool to be used within the complex environment of Earth system models to speed up specific model components or to improve the representation of processes that cannot be represented adequately by physical equations. The use of NNs in the development of parameterisation schemes may also enable new approaches for representing model uncertainty in ensemble predictions.

Today many scientific groups around the world are trying to answer the more general question that is controversial: can forecast models that are based on deep learning and trained on atmospheric data compete with or even beat weather and climate models that are based on physical knowledge and the basic equations of motion? Given the increasing number of meteorological observations that are available to train NNs, in particular since the beginning of the satellite era, the use of NNs may not be limited to parameterisation schemes and specific model components in the future. NNs may also come to compete with existing weather forecast models as a whole to perform actual weather predictions if observations of the past are used for training while observations of the present are used as input to generate forecasts. Global weather forecast models that solve three-dimensional, non-linear equations may become obsolete. It can be assumed that more observations can be used for predictions in a weather forecast system based on NNs in comparison to predictions with dynamical models since data preprocessing and selection could be done by the networks and since higher resolution can be used for predictions since NNs can be expected to be much cheaper and easier to optimise for HPC in comparison to conventional models.

NNs have been used to generate local weather predictions (see, for example, Hall et al., 1999), and it has been shown that NNs can be used to improve El Niño predictions of au- toregressive integrated moving average techniques (Nooteboom et al., 2018). However, it has yet to be shown that forecasts based on deep learning can compete with global operational weather forecast models, in particular in the medium range. Answering this question is difficult since it requires scaling up the training process of NNs to the level of complexity of a large supercomputing application to allow a fair comparison between the two approaches. In this paper we will make the first step and discuss the potential of $\mathrm{NNs}$ for global weather predictions. We base this discussion on tests with NNs that are used to represent the equation of the Lorenz'95 model - which is a low-complexity model to test new approaches to atmospheric modelling - as well as a NN toy model for global weather predictions that is trained on atmospheric reanalysis data. In both cases, no dynamical equations are used to update the model state. Results will be used to identify challenges and fundamental design choices for a forecast system based on NNs. Tests with Lorenz'95 serve as an example for a system for which the basic equations are known while the exact equations are unknown for the toy model for global weather predictions.

Our tests with Lorenz'95 and the toy model for global weather forecasts are presented in Sect. 2. Based on the results, Sect. 3 discusses challenges and fundamental design choices for the development of forecast systems based on NNs. Section 4 presents the conclusions.

\section{Results with neural networks}

Section 2.1 will present results for initial tests with the Lorenz'95 low-complexity model that serves as a test bed for atmospheric dynamics. We will then develop a toy model for the global atmosphere that is used to calculate global weather forecasts in Sect. 2.2.

NNs consist of neurons that resemble properties of neurons in the brain in terms of functionality and connectivity. In NNs, neurons are connected with each other and organised in layers. All networks that are used in the following are sequential NNs that use a linear stock of layers of neurons for which each neuron is connected to each neuron of the previous and subsequent layer, a so-called multilayer perceptron (MLP). Information travels from the inputs in the first layer to subsequent layers. The outputs will leave the NN in the output layer. The layers between the input and output layers are called hidden layers. Each neuron is a weighted sum over all inputs (all neurons of the previous layer) plus a bias term $\left(\sum_{i=1}^{N} w_{i} n_{i}+b ; w_{i}\right.$ are weights, $b$ is the bias term). An activation function is applied to the accumulated value to represent non-linearity. During the training phase of the NN, the weights and biases within the network are optimised by reducing a loss function (mean absolute error for results in this paper). We use the Keras Python library (Chollet et al., 2015) to train and apply NNs for all results that are presented in the following. We have tested several activation functions 
and optimisers and obtained the best results using hyperbolic tangents as a activation function and a stochastic gradient descent optimiser. Overall, $20 \%$ of the training data is used for validation during training. All input and output data are normalised. All NNs that are used were trained for at least 200 training iterations that go through the entire dataset during optimisation, called epochs.

\subsection{Initial tests with a toy model for atmospheric dynamics}

We study the three-level Lorenz'95 model that was presented in Thornes et al. (2017). The model extends the original twoscaled Lorenz' 95 model (Lorenz, 2006) by one more level such that it provides flexibility regarding tests at different resolutions in a toy model for atmospheric dynamics. The model consists of three levels of model variables that can be assumed to be large-scale " $X$ ", medium-scale " $Y$ " and small-scale " $Z$ ". At each level, all degrees of freedom form a one-dimensional ring, and the number of degrees of freedom increases by a factor of 8 from one level to the next ( 8 for $X, 64$ for $Y, 512$ for $Z$ ). Eight degrees of freedom on a finer model level are coupled to 1 degree of freedom on the coarser level respectively. The degrees of freedom are described by the following differential equations:

$$
\begin{aligned}
\frac{\mathrm{d} X_{k}}{\mathrm{~d} t}= & X_{k-1}\left(X_{k+1}-X_{k-2}\right)-X_{k}+F-\frac{h c}{b} \sum_{j=1}^{J} Y_{j, k}, \\
\frac{\mathrm{d} Y_{j, k}}{\mathrm{~d} t}= & -c b Y_{j+1, k}\left(Y_{j+2, k}-Y_{j-1, k}\right)-c Y_{j, k}+\frac{h c}{b} X_{k} \\
& -\frac{h e}{d} \sum_{i=1}^{I} Z_{i, j, k}, \\
\frac{\mathrm{d} Z_{i, j, k}}{\mathrm{~d} t}= & e d Z_{i-1, j, k}\left(Z_{i+1, j, k}-Z_{i-2, j, k}\right)-g_{Z} e Z_{i, j, k} \\
& +\frac{h e}{d} Y_{j, k} .
\end{aligned}
$$

The indices $i, j$ and $k$ range from 1 up to $I=8, J=8$ and $K=8$ for the $Z, Y$ and $X$ tiers. $F$ is a large-scale forcing term which determines the chaoticity of the model and is set to 20 in the simulations of this paper, which results in fully chaotic model dynamics. The remaining parameters allow tuning of the frequency and amplitude of oscillation as well as the coupling between tiers and are set to $h=1$, $c=b=e=d=10$ and $g_{Z}=1$ to obtain slow oscillations with a large amplitude in the $X$ tier while the other two tiers oscillate more quickly at a lower amplitude. A fourth-order Runge-Kutta method is used to integrate the model in time using a time step of 0.005 model time units (MTUs).

We consider a model simulation that uses all of the scales as a "truth". Similar to a weather prediction model that truncates spatial resolution, we can mimic limited resolution in the Lorenz'95 model by truncating the medium- and smallscale degrees of freedom.

We have trained several NNs to predict the tendency of the model $\left(\Delta X_{k}^{n}=X_{k}^{n+1}-X_{k}^{n}\right)$ to update the state vector in one time step. The NNs are used iteratively to make predictions for more than one time step $\left(X_{k}^{m+1}=X_{k}^{m}+\Delta X_{k}^{m}\right)$. To reduce the error due to time discretisation, the calculation of the right-hand-side tendency via the NN is coupled to a third-order Adams-Bashforth explicit time-stepping scheme. We use a first- and second-order scheme for the first two time steps. The medium- and small-scale variables $\left(Y_{j, k}\right.$ and $\left.Z_{i, j, k}\right)$ are not represented in the $\mathrm{NN}$, neither as inputs nor as outputs.

We use two different architectures to set up forecast models. A "global" approach uses all eight $X_{k}$ variables as input to predict the tendencies. On the other hand, a "local" approach uses $X_{k-2}, X_{k-1}, X_{k}$ and $X_{k+1}$ as input to predict a tendency for a single variable $X_{k}$. For the local approach, the same NN is called eight times to update the entire state vector. The pairs of training sets are separated by one model time unit in the truth run to generate data points that are sufficiently uncorrelated. We use 2000000 pairs of $X_{k}^{m+1}$ and $X_{k}^{m}$ to train the NNs. For local NNs the information for each variable $X_{k}$ of a training set with a full state vector is used as an independent training set.

We have performed tests with many different NN configurations with different layer width and number of layers. We achieved the best results using four hidden layers between the input and output layer with 100 neurons per layer for the global setup and two hidden layers with 20 neurons each for the local setup. These configurations were used in the following.

Figure 1 shows results for the two NNs. The trajectories that are calculated with the NNs show the typical dynamic of a Lorenz'95 model, and it is indeed possible to generate reasonable forecasts with the two models based on NNs. The error of the local NN configuration is significantly lower compared to the global configuration. For comparison, we mimic a standard forecast with limited resolution using a dynamic model that is based on Eq. (1) (see Fig. 1b). The medium- and small-scale variables $\left(Y_{j, k}\right.$ and $\left.Z_{i, j, k}\right)$ are not represented. No sophisticated parameterisation scheme is used and the coupling terms for the degrees of freedom that are not resolved are removed with no replacement (the last terms in Eq. 1). The standard model produces a lower forecast error compared to the global NN forecast systems and a lower forecast error for the local $\mathrm{NN}$ forecast system at the beginning of the forecast. However, the error for the local NN forecast system is lower towards the end of the forecast.

\subsection{A toy model for global weather forecasts}

We have developed a toy model to simulate the dynamics of the global atmosphere that can be used for global weather forecasts. We have focussed on the representation of geopo- 

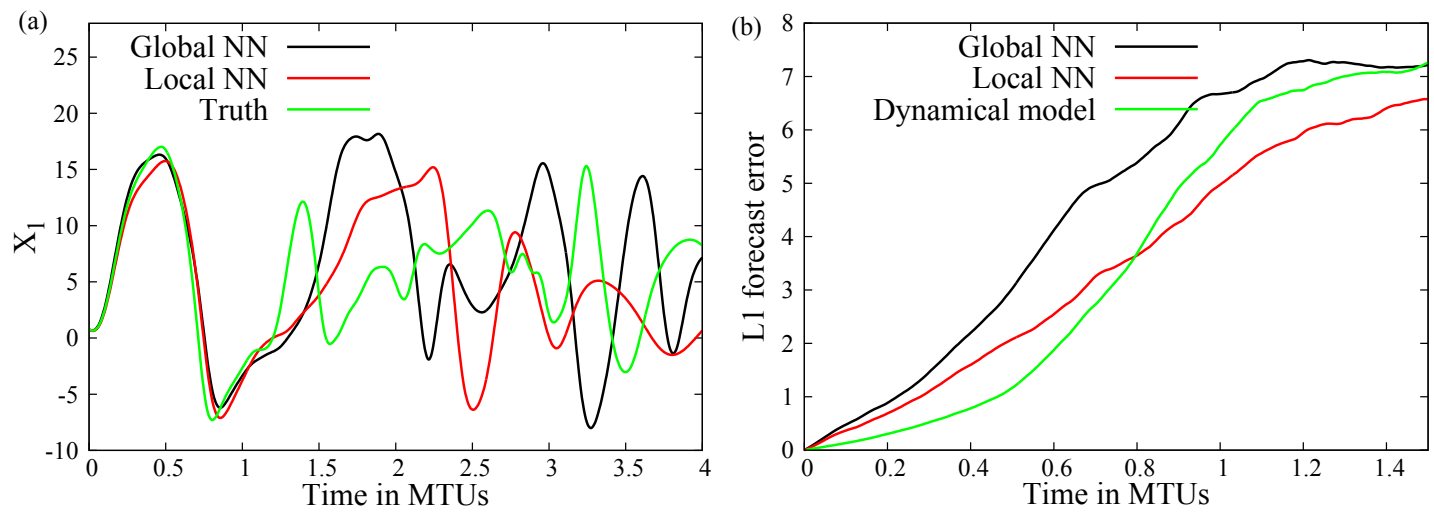

Figure 1. (a) Example trajectory for the global and local NN as well as the truth. (b) L1 error for forecasts with the global and local NN as well as the truncated dynamical forecast model.

tential height at $500 \mathrm{hPa}(\mathrm{Z} 500)$, which is a standard field for analysing the quality of weather forecasts. Z500 was picked since the dependency on local conditions such as topography is limited (in contrast to fields such as surface pressure), since Z500 is not spotty with very strong local gradients (in contrast to fields such as humidity or precipitation) and since most of the important global flow pattern - such as midlatitude jets and a gradient between poles and Equator - are visible.

\subsubsection{General model setup}

We use Z500 data of the ERA5 reanalysis dataset (ERA, 2018) for training. Reanalysis data are the state of an atmospheric model with a continuous assimilation of observations to generate the best possible picture of the global atmosphere at a given time. The advantage of the use of a reanalysis dataset instead of observations for training is that data are available for each grid point at each time step and that the data are consistent over the entire data window. However, the use of analysis data restricts the use of the NN model to the forecast only. A conventional data-assimilation system is still necessary to generate initial conditions.

We use hourly data of Z500 from ERA5 for training and map the data to a longitude-latitude grid with $6^{\circ}$ resolution. We therefore consider global snapshots of Z500 with $60 \times$ $31=1860$ grid points. Overall, 67200 of these snapshots are available in total in the period between 1 January 2010 and 31 August 2017.

We have tested many different $\mathrm{NN}$ architectures and training configurations. Some changes to the setup had a significant influence on the quality of the toy model. We will only present the most successful approaches in the following. We have played around with normalised fields that would use the anomaly field and remove the annual mean from the data. However, we found that the use of absolute field values achieved the best results. Similar to the NNs for the Lorenz model, all NNs are trained with Z500 data at the full hour $(n)$ to predict the tendency as the difference in $\mathrm{Z} 500$ $\left(\triangle Z 500=Z 500^{n+1}-Z 500^{n}\right) 1 \mathrm{~h}$ later $(n+1)$. This tendency is equivalent to the right-hand side of a differential equation in time if the time step is $1 \mathrm{~h}$. The NNs are used iteratively with a third-order Adams-Bashforth explicit time-stepping scheme to make predictions for more than $1 \mathrm{~h}$ into the future.

Time was used as additional input variable for all NNs. There is one coordinate that represents the daily cycle (growing linearly from midnight to midnight of the following day) and one coordinate to represent the annual cycle growing linearly from the beginning to the end of the year with a correct representation of leap years.

In a second set of tests, we have included $2 \mathrm{~m}$ temperature $(2 \mathrm{mT})$ as an additional prognostic field in the $\mathrm{NN}$ forecast system. ERA5 data for $2 \mathrm{mT}$ were retrieved and processed in the same way as for Z500 and added as additional input and output parameters.

\subsubsection{Global and local networks}

As for the Lorenz case, we present results for a global and a local model configuration. Global networks are using all $60 \times 31$ grid points plus the two time coordinates as input to calculate the $60 \times 31$ tendencies for each grid point that are used to update the entire state vector $1 \mathrm{~h}$ into the future. This process is iterated to make predictions for longer lead times. Local networks are using a stencil of $N \times N$ points to calculate the tendency for a single grid point in the centre of the stencil, with $N$ being an odd integer number. This is similar to finite-difference schemes in conventional models. We have trained NNs for different stencil sizes for the input fields $(3 \times 3,5 \times 5,7 \times 7,9 \times 9)$. For each stencil size, the same $\mathrm{NN}$ is used to update grid points in the entire domain. However, to enable the $\mathrm{NN}$ to learn and represent local dynamics, we have added the horizontal coordinates longitude and latitude as additional input variables. The approach of a local stencil is easy to realise, but it generates a standard problem for all 
forecast models that are based on longitude-latitude grids: the pole requires special treatment.

To represent polar areas in local networks we have trained a special NN to update the north and south pole of the grid. The poles are represented by the first and last latitude band of the grid ( 60 grid points for each pole but all of the points have the same value). The special pole NN was trained with the value of the pole plus the 60 variables of the next latitude band as input to output the tendency to update the pole. If we use a stencil of $5 \times 5$ grid point inputs to update the bulk of the grid points, we use the special pole NN to update the poles, a $3 \times 3$ stencil $\mathrm{NN}$ to update the latitude bands that are closest to the poles and the $5 \times 5$ stencil $\mathrm{NN}$ for all other points. To use a $9 \times 9$ stencil NN would require the use of a $7 \times 7$, a $5 \times 5$ and a $3 \times 3$ stencil $\mathrm{NN}$ as well as the special polar NN towards the two poles.

We have tested many different configurations of the NNs to identify the setups that produced the best results. For the results of the following section, we used four hidden layers of neurons that have the same width as the input layer (1862 neurons for the global and $N \times N+4$ neurons for local configurations). For the NNs that use both Z500 and 2mT as input, the same configuration for neurons in hidden layers was used for the local configurations while the width of the hidden layers was increased to the size of the new input vector for the global configuration and the number of hidden layers was reduced to two.

\subsubsection{Results}

Figure 2 shows the global forecast error compared against the analysis that is used for operational forecasts at ECMWF for the local NN configuration and different stencil sizes. The forecast error was calculated as the average of 10 forecasts distributed equally between March 2017 and February 2018. Some of the dates that have been used to calculate the forecast error are also used when training the networks. However, this overlap is not a problem since a different analysis dataset has been used for initialisation. The forecast error is not very different for different stencil sizes at the beginning of the forecast. However, for the local networks that use the special treatment of the area around the pole, as discussed in the previous section, the forecast error diverges for the $7 \times 7$ and the $9 \times 9$ configuration. When looking into the actual fields, it became visible that this divergence is caused by chequerboard patterns developing close to the poles. We therefore started a second set of simulations that used the original stencil size but kept all grid points that could not be calculated with the largest stencil fixed throughout the forecast. These simulations remained stable for much longer lead times of up to around 2 weeks, and it is visible that the lines do not diverge in the same way towards the end of the forecast. The difference in forecast error between the two approaches is very small at the beginning of the forecasts.
It is likely that the instabilities that were found near the poles could be removed using a polar filter or a relaxation of the model fields against a reference solution. These methods have been used to stabilise conventional grid point models near the poles in the past. It is also possible that the pole problem can be solved via a change in the network architecture of the NNs that are used in the vicinity of the poles. However, a more detailed investigation of this problem is beyond the scope of this paper.

Figure 3a shows the same global forecast error as Fig. 2 when using different methods to generate the forecasts. We show results for forecasts with the Integrated Forecast System (IFS) at very coarse resolution (TL21 with 60 vertical levels) as well as operational forecasts. The TL21 resolution forecast uses a coarser horizontal resolution, and model data were mapped to the $6^{\circ}$ longitude-latitude grid to calculate the forecast error. Therefore, the initial error is not zero for the TL21 forecast. We also show the persistence forecast error when assuming that the Z500 field will not change during the forecast window. The forecasts with local NNs beat TL21 forecasts at the beginning of the forecast as well as the persistence forecasts during the full 5-day period. The global network shows little benefit compared to the persistence forecast.

Figure $3 \mathrm{~b}$ shows the global forecast error for Z500 when $2 \mathrm{mT}$ was added as prognostic field in the forecast system. The quality of forecasts for Z500 did not improve in comparison to the forecast system based on Z500 only.

Figure 4 shows the Z500 fields for a 1-day forecast with a single field as well as the change in Z500 during the first day of the forecast with the local and global NN configuration. The analysis that is used for operational forecasts at ECMWF is also presented. The Z500 fields that are generated with the NNs look healthy and reasonable after 1 day with no obvious problems in the solution. Generated videos that visualise the development of the field in time (attached as a Supplement) also look very realistic for the first couple of days. The difference between the observed tendencies and the local network is small during the first forecast day. The global network seems to underestimate the magnitude of changes.

\section{Challenges and fundamental design choices for forecast systems based on NNs}

The results of the previous section show that it may indeed be possible to generate global weather predictions based on NNs for short-range prediction and at this rather course spatial resolution. Whether a NN prediction system will ever be competitive with state-of-the-art weather prediction models remains an open question. It would certainly require a serious level of complexity with as many (or more) degrees of freedom as conventional models. To develop such a system, a couple of important decisions need to be made regarding 

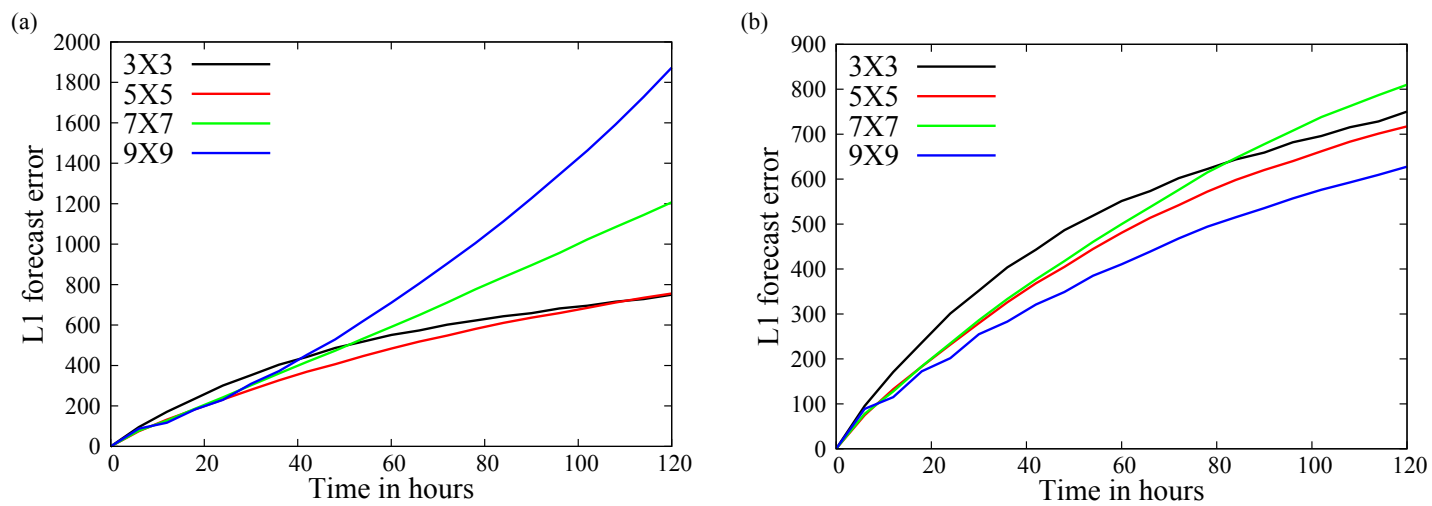

Figure 2. Globally integrated absolute forecast error plotted against time for local NNs with different stencil size. (a) NNs that use local networks with smaller stencil size as well as the special pole network towards the poles. (b) NNs that fix the fields close to the poles to stabilise simulations.

(a)

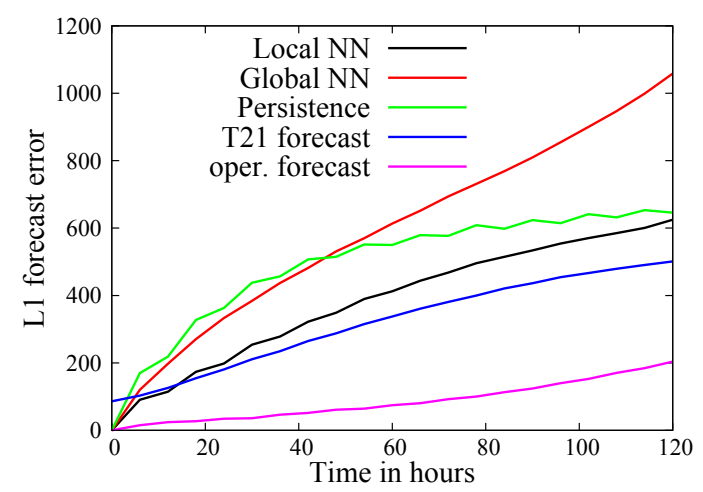

(b)

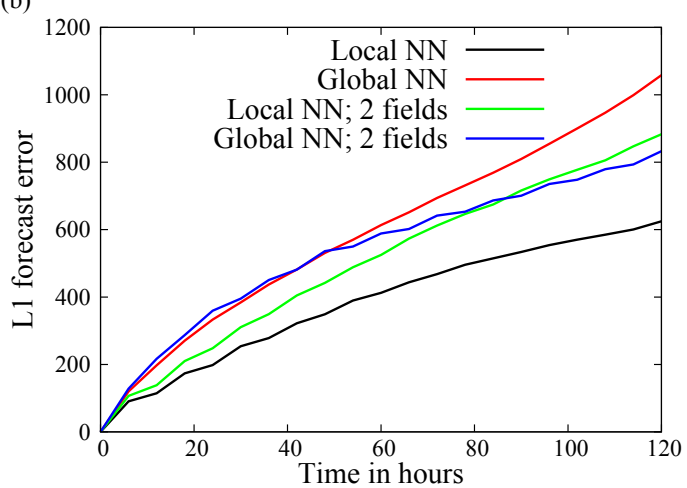

Figure 3. (a) Globally integrated absolute forecast error for the best local network $(9 \times 9$ stencil), the global network, a persistence forecast, an IFS forecast at TL21 resolution and the operational weather forecast of ECMWF. The persistence forecast shows a 12-hourly fluctuation since Z500 has a weak 12-hourly cycle in the tropics due to atmospheric tides. (b) The same globally integrated absolute forecast error for the best local and global network as in (a) plus the best results for local and global networks that use $2 \mathrm{mT}$ as additional prognostic field.

the structure of the networks and the shape of the training data. We can only guess how the optimal configuration of a NN prediction system may look. This section will identify and discuss some of the important challenges and design choices.

\subsection{Local or global networks and time-stepping schemes}

Should forecasts with NNs be iterative or should they be trained for specific lead times? Should global forecasts be performed by a single NN that takes as many data inputs as possible or would it be better to use many NNs that are coupled together?

State-of-the-art weather forecast models use up to a billion variables to represent the state of the Earth system. Atmosphere and ocean show chaotic behaviour with scale interactions that result in exponential error growth. Global weather forecast models have skill for several forecast days into the future and can be used for seasonal predictions. This requires models to be able to represent complex interactions between weather features, such as convection in the tropics that generates gravity waves to influence jet position in the midlatitudes. To enable forecast systems based on NNs to be competitive in global weather predictions several days into the future would require them to (1) represent all relevant scales and features all over the globe, (2) allow scale interactions and (3) be able to represent chains of complex interactions between weather features.

Similar to the use of explicit or implicit time-stepping methods for conventional models, there seem to be two approaches to achieve (1)-(3) with NNs. Either all information is connected, such as in implicit methods, to allow large time steps, or the connectivity of networks is local and time steps are short, such as in explicit time-stepping schemes. The sheer size of the Earth will make it difficult to connect all information in global networks, and if global all-to-all commu- 

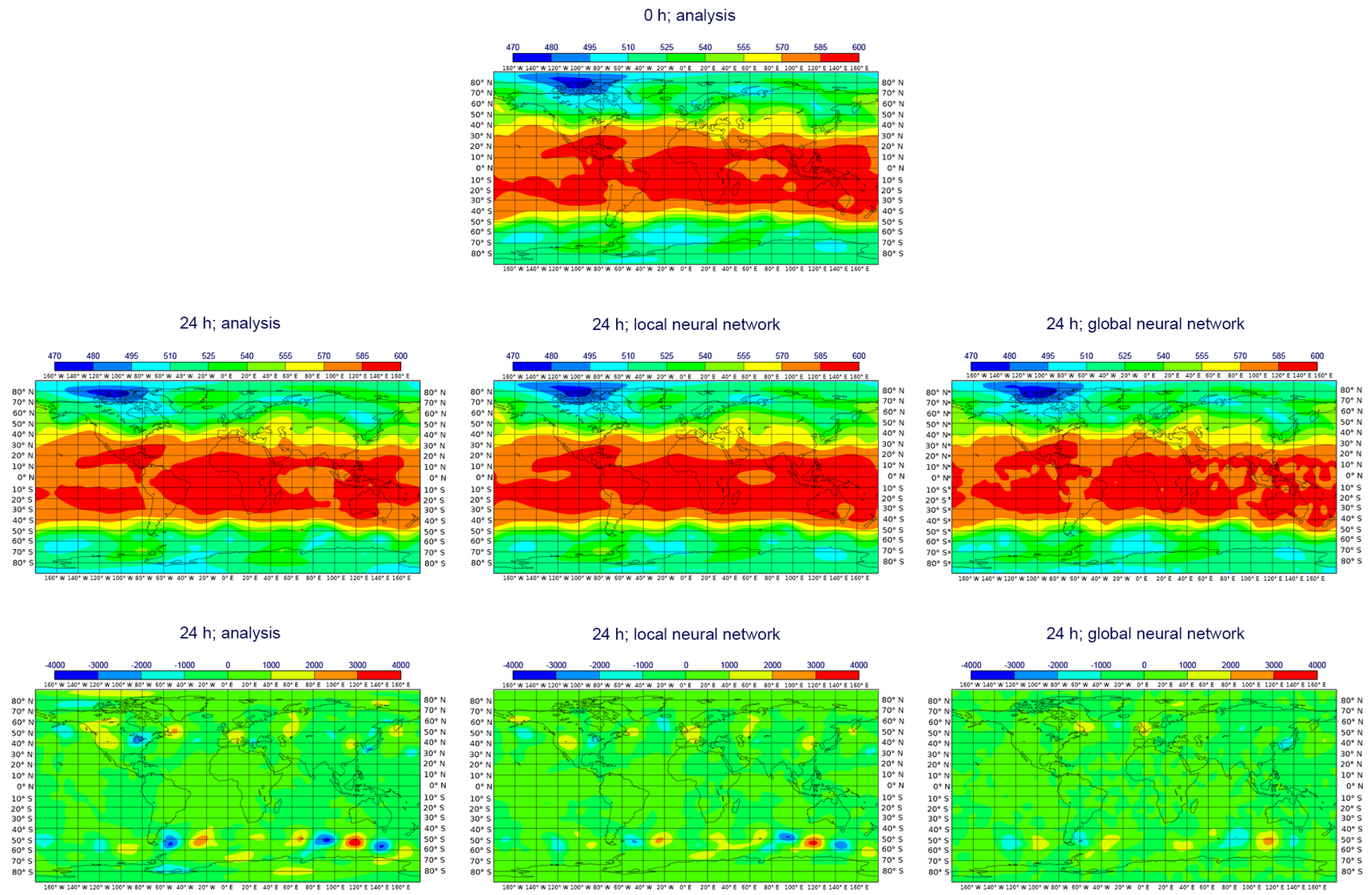

Figure 4. Top: Z500 from analysis on 1 March 2017 that is used as initial conditions for forecasts. Middle: Z500 for the analysis on 2 March for reference (left) as well as the local network and the global network configurations 1 day into the forecast (middle and right). Bottom: difference between Z500 for the analysis on 1 and 2 March (left) as well as the difference between initialisation and after $24 \mathrm{~h}$ for the local and the global network configuration (middle and right). The local network uses a $9 \times 9$ stencil and fixed polar regions.

nication across many compute nodes of an HPC system were required, NNs would loose much of their performance benefit in comparison to conventional models on modern HPC facilities. High-resolution global networks could certainly not be dense, meaning that all neurons between different layers are connected as in MLPs. The global networks at very coarse resolution that were used in the paper already use a very large number of tunable parameters in comparison to NNs used in other disciplines due to their dense character. To reduce the amount of trainable parameters with no loss in scale interactions will be important.

On the other hand, local networks would need to be applied in iterative ways to allow interactions between scales and features in predictions for longer lead times. It can be argued that the lead time needs to be adjusted to the size of the local stencil of inputs. If local networks are used, there is another decision to be made: should the same network be used for all grid points or should different networks be used for different locations around the globe. In this paper, we use the same NN for all grid points. However, an approach that used a different network for different locations could be realised using convolutional networks that combine stencil information locally within one of the layers but do not propagate information throughout the entire grid. This would allow us to update a large part of the domain with a single network and to exploit standard network configurations for image processing, at least if structured grids are used. However, it would require significant work to make sure that boundary conditions are represented correctly. This approach was therefore not tested in this paper.

Can we assume that NNs can use time steps that are as long or longer than time steps of implicit time-stepping schemes in conventional models? This should be possible in principle since a complex network with global internode communication could, in an extreme case, resemble an implicit time step of a conventional model one-to-one. However, the amount of training data and network complexity that is necessary will quickly become prohibitive if interactions between more and more features and longer chains of causality are to be represented.

Results with our toy model suggest the following: 
- It is fundamentally possible to generate global weather forecasts when connecting iterative time-stepping schemes with NNs. This conclusion is entirely based on very coarse resolution data and derived from assessing the predictive skill for a variable that varies much more slowly in space and time than, for example, precipitation. However, none of the network configurations that we tested allowed stable simulations for integrations of more than 2 weeks into the future, and forecast skill quickly deteriorates after a couple of days. If networks are applied iteratively, it will be important to satisfy fundamental conservation properties and to stabilise simulations. This is non-trivial and will require attention, in particular if it is the aim to generate climate predictions. It is also likely that model biases - that are difficult to distinguish from forecast variance - will perturb predictions of a $\mathrm{NN}$ forecast system.

- It will be difficult to train networks to make predictions with long lead time with a single time step. When we tried to train NNs to make prediction with longer time steps than $1 \mathrm{~h}$, results were degraded significantly. A linear reduction in the time step will most likely be required if resolution is increased, similar to Courant-Friedrichs-Lewy (CFL) condition requirements for conventional models.

- Results are much better for the local approach when compared to the global approach for both the Lorenz'95 model and the toy model for global predictions. We decided to use the same network to update grid points all over the domain instead of using special networks for each grid point. This approach appears more promising since the resulting network is more likely to be consistent with physical laws since it is trained for many different physical situations. Local information can be represented even if the network is used in the entire domain when adding spatial coordinates to the inputs. If a customised network is used for each grid point or if a convolutional network is used to connect local stencils, the amount of data that are available for training is reduced by the number of grid points, and the representation of extreme events within the training data will often be questionable, in particular in a changing climate.

\subsection{Understanding the physical system}

How far can we go using a "black box"? NNs allow us to solve non-linear systems as a black box with no knowledge of the actual physical system. There is no reason why weather forecasts could not be generated with such a black box. However, the work on the toy model clearly indicated that a physical understanding is still important to improve the $\mathrm{NN}$ architecture and training to perform weather predictions.

One example is the time-stepping scheme that was used. In a first approach, we have tried to generate daily or 6- hourly forecasts with a single step with only very limited success. When thinking about possible problems, we remembered that an explicit time-stepping scheme would require much shorter steps at the given level of model resolution. Due to our knowledge of the level of numerical complexity that is required to allow weather and climate models to run at longer time steps, we concluded that it would be much easier for the NN to learn the correct dynamics for shorter time steps and switched the dataset from ERA Interim (Dee et al., 2011) to ERA5 to allow training with hourly data. Results improved significantly.

A second example relates to the preprocessing of input and output data. Meteorological data come in very different shapes. For example, while specific humidity seems to be an easy quantity since it is confined to between 0 and 1 , very small values can still be very important and values can change by orders of magnitude between the stratosphere and the troposphere within a single vertical level; precipitation can have very significant outliers in extreme events; fields such as geopotential height or temperature show global gradients and local features which makes it difficult to judge whether gradients or absolute fields are important; fields such as surface pressure depend heavily on local features such as topography. If these fields are used as input or output for NNs to predict weather, physical knowledge of the properties of the fields and their connectivity will be essential to design the optimal data preprocessing and the optimal network architecture. It may be possible to heal shortcomings of data preprocessing and network configurations with a brute-force increase in data volume and training time as long as limits in numerical precision do not remove information from data. However, success will be limited, in particular since many trial and error tests at large computational cost will be necessary to find the optimal network configuration for complex configurations that have the ambition to compete with conventional weather forecast models.

At the given state of software and science it is very difficult for domain experts to evaluate and understand connectivity of data in NNs to be in a good position to improve predictions. Networks are treated as a black box. The case of the Lorenz' 95 model is a good example. If sub-grid-scale parameters are ignored for the moment, the differential equations and correct connectivity between the prognostic variables are known. It should be possible to design and train NNs that resemble the exact behaviour of the equation and compete with the dynamic model. Since parameters are adjusted to the data, networks should also be able to automatically incorporate parameterisation schemes for sub-grid-scale variables and to beat dynamic models that do not have parameterisation schemes. However, it was unclear how to project our knowledge onto the design of the $\mathrm{NN}$ and the training procedure. As a consequence, we did not manage to obtain results that were close to optimal. However, we do not claim that we have tested all possible options. While it is likely that even small changes in the network setup may have im- 
proved results significantly, the parameter space that needs to be explored to find the optimal configuration has many dimensions (activation function, \#layers, \#neurons, \#epochs, \#datasets, data normalisation, connectivity between neurons, ...). This makes the search for the optimal configuration cumbersome. Tests with $2 \mathrm{mT}$ as an additional field in the global toy model also showed that it is difficult to relate different model fields to each other and that it is not sufficient to just add input information to improve predictions. A physical understanding of connectivity and signal propagation is much easier in conventional models that allow us to assign a physical meaning to all parameters. To develop tools and approaches for domain scientists to understand and improve connectivity within complex NNs will be essential.

\subsection{Data}

What data should be used for training and how should they be preprocessed? How can observational data be assimilated to generate initial conditions for forecasts?

A vast amount of meteorological data are available that could be used for training of forecast models. The data handling system of ECMWF provides access to over $210 \mathrm{~PB}$ of primary data, and the data archive of ECMWF grows by about 233 TB per day (ECM, 2018). However, it is questionable how much of this data can actually be used for training, and the numbers shrink substantially depending on choices regarding network architecture and the selection of training data.

If unfiltered observational data are used as input for networks, biases between different observation systems need to be addressed and networks need to be robust against missing or faulty input data. In a first step, it would probably be necessary to use data from a small number of sources that provide a continuous spatial coverage rather than a moving data window. Even if, for example, all satellite data could be used for training, such data would only be available for a couple of decades.

It is also questionable how much data can actually be used given that the climate of the Earth is constantly changing and in particular given the rapid changes due to anthropogenic climate change. This will also decrease the time frame for which data can be used for training since we cannot expect a model that is trained as a black box to provide reliable predictions if the underlying climate state has changed and if events that have never happened in the training data start to happen in the real world, such as an ice-free Arctic during summer. Conventional models show significant biases in long-term simulations. These biases will also be a problem for models based on NNs and may change the local "climate" within a couple of days of simulations and push the network to weather regimes that were not covered by the training data.

For a model that is based on physical principles, it can be assumed that changes in the general circulation or the frequency and shape of extreme events due to climate change can be represented correctly, at least within limits. Local networks that are trained to represent the dynamics at all grid points, as in the toy model, will be better suited for simulations in a changing climate compared to networks that are trained for local conditions at each grid point since they have been trained in the context of a different local climate.

Existing weather and climate models could be used to generate a shear unlimited amount of training data to train a forecast system based on NNs also for a changing climate. Reanalysis data that were used for the toy model could be used as well. However, the quality of the existing models and the assimilation system would limit the quality of predictions with the $\mathrm{NN}$ forecast system, reducing the advantage of the deep-learning approach. Tests with the toy model clearly indicate that it will require data with very high temporal resolution to develop a NN forecast system that can run at high resolution, similar to the length of time steps in conventional models, which will not be available in standard reanalysis datasets or for standard long-term model integrations and require a very large data system.

The results with the toy model suggest that it is difficult enough to develop forecast models if consistent data with full spatial coverage are used for training. This suggests starting investigations towards a $\mathrm{NN}$ forecast system with reanalysis or model data which is less noisy and more consistent when compared to real-world observations. If it is possible to replicate the dynamics of the model in sufficient detail, more difficult tasks such as the use of real observations as input can be addressed.

\section{Conclusions}

We have developed a toy model based on NNs to generate global weather forecasts. The toy model does not use any dynamical equation of motion. The model is based on a $6^{\circ}$ longitude-latitude grid and represents Z500 as the only model field. We show that it is indeed possible to make predictions into the future that are better than a simple persistence forecast and are competitive with forecasts of very coarse-resolution (TL21) atmosphere models of similar complexity at least for short lead times. We did not intend to build a model that can be competitive with operational weather forecast models. However, we use the toy model to identify challenges and to indicate fundamental design choices that may lead to optimal results for forecast systems based on NNs.

While the use of deep-learning techniques is often discussed as being a silver bullet to represent non-linear systems, it has yet to be shown whether weather forecast systems based on NNs can be competitive compared to conventional models in particular for global forecasts, for longer time ranges than 1 day and across the wide range of physical parameters that are provided by numerical models with physical consistency. The experience with the toy model suggests 
that there will be no free lunch. While NNs can, in principle, be used as a black box, the development of a weather forecast system will require domain knowledge about the Earth system. Close collaborations between computer scientists and meteorologists will be essential even if petabytes of training data and exascale supercomputers are available. A deep understanding of how to use physical knowledge of the Earth system and the connectivity between degrees of freedom to improve the development of network architectures and network training and how to preserve conservation properties will be required.

The development of a NN forecast model that is based on a model grid as discussed in this paper will face similar challenges when compared to the development of conventional models such as the complexity of the Earth system with nonlinear interactions between model components, scale interactions, exponential growth of errors in initial conditions, numerical instabilities and the discrete representation of model fields on the sphere (leading to the pole problem in our toy model), the treatment of conservation properties, model biases in long-term simulations, errors in observations, and insufficient data coverage of observations. On the one hand, it is likely that NN models could make better use of future computing hardware and use more observations and higher resolution. On the other hand, it is also likely that it will be difficult to stabilise long-term integrations (in particular in a changing climate) and to represent complex interactions between model features in long simulations since it will be hard to improve physical consistency within networks. For $\mathrm{NN}$ forecast systems that try to describe the evolution of the atmosphere based on grid points after learning the right-hand side of the equations of motion from data, it therefore seems obvious to focus on short-term forecasts and potentially also regional predictions in a first approach. It will be difficult for these models to compete with conventional models in medium- and long-range weather forecasts and climate predictions. However, the same statement may not be true for other applications of NNs that do not propagate the full atmosphere to make long-term forecasts but rather focus on predictions of large-scale flow patterns such as El Niño and weather regimes (see, for example, Nooteboom et al., 2018).

Code availability. The code that was used to generate the result of this paper is attached as a Supplement.

Data availability. Only standard ERA5 reanalysis data (see ERA, 2018) were used for training.

The Supplement related to this article is available online at https://doi.org/10.5194/gmd-11-3999-2018supplement.
Author contributions. The paper was written by both authors. Numerical experiments were done by PDD.

Competing interests. The authors declare that they have no conflict of interest.

Acknowledgements. Peter D. Düben gratefully acknowledges funding from the Royal Society for his University Research Fellowship and the ESIWACE project. The ESIWACE project has received funding from the European Union's Horizon 2020 research and innovation programme under grant agreement no. 675191. Many thanks to Christoph Angerer, John Griffith, Peter Watson and Nils Wedi for very helpful comments and advice.

Edited by: David Topping

Reviewed by: two anonymous referees

\section{References}

Brenowitz, N. D. and Bretherton, C. S.: Prognostic Validation of a Neural Network Unified Physics Parameterization, Geophys. Res. Lett., 45, 6289-6298, https://doi.org/10.1029/2018GL078510, 2018.

Chevallier, F., Chéruy, F., Scott, N. A., and Chédin, A.: A Neural Network Approach for a Fast and Accurate Computation of a Longwave Radiative Budget, J. Appl. Meteorol., 37, 1385-1397, https://doi.org/10.1175/15200450(1998)037<1385:ANNAFA>2.0.CO;2, 1998.

Chevallier, F., Morcrette, J.-J., Chéruy, F., and Scott, N. A.: Use of a neural-network-based long-wave radiative-transfer scheme in the ECMWF atmospheric model, Q. J. Roy. Meteor. Soc., 126, 761-776, https://doi.org/10.1002/qj.49712656318, 2000.

Chollet, F. et al.: Keras, available at: https://github.com/fchollet/ keras (last access: 28 September 2018), 2015.

Dee, D. P., Uppala, S. M., Simmons, A. J., Berrisford, P., Poli, P., Kobayashi, S., Andrae, U., Balmaseda, M. A., Balsamo, G., Bauer, P., Bechtold, P., Beljaars, A. C. M., van de Berg, L., Bidlot, J., Bormann, N., Delsol, C., Dragani, R., Fuentes, M., Geer, A. J., Haimberger, L., Healy, S. B., Hersbach, H., Hólm, E. V., Isaksen, L., Kållberg, P., Köhler, M., Matricardi, M., McNally, A. P., Monge-Sanz, B. M., Morcrette, J. J., Park, B. K., Peubey, C., de Rosnay, P., Tavolato, C., Thépaut, J. N., and Vitart, F.: The ERA-Interim reanalysis: configuration and performance of the data assimilation system, Q. J. Roy. Meteor. Soc., 137, 553-597, https://doi.org/10.1002/qj.828, 2011.

ECMWF: Homepage, available at: https://www.ecmwf.int/en/ computing/our-facilities/data-handling-system, last access: 16 June 2018.

ERA5: dataset, available at: https://www.ecmwf.int/en/forecasts/ datasets/reanalysis-datasets/era5, last access: 10 April 2018.

Gentine, P., Pritchard, M., Rasp, S., Reinaudi, G., and Yacalis, G.: Could Machine Learning Break the Convection Parameterization Deadlock?, Geophys. Res. Lett., 45, 5742-5751, https://doi.org/10.1029/2018GL078202, 2018.

Hall, T., Brooks, H. E., and Doswell III, C. A.: Precipitation Forecasting Using a Neural Network, Weather 
Forecast., 14, 338-345, https://doi.org/10.1175/15200434(1999)014<0338:PFUANN>2.0.CO;2, 1999.

Krasnopolsky, V. M. and Fox-Rabinovitz, M. S.: A new synergetic paradigm in environmental numerical modeling: Hybrid models combining deterministic and machine learning components, Ecol. Model., 191, 5-18, https://doi.org/10.1016/j.ecolmodel.2005.08.009, 2006.

Krasnopolsky, V. M. and Lin, Y.: A Neural Network Nonlinear Multimodel Ensemble to Improve Precipitation Forecasts over Continental US, Adv. Meteorol., 2012, 649450, https://doi.org/10.1155/2012/649450, 2012.

Krasnopolsky, V. M., Chalikov, D. V., and Tolman, H. L.: A neural network technique to improve computational efficiency of numerical oceanic models, Ocean Model., 4, 363-383, https://doi.org/10.1016/S1463-5003(02)00010-0, 2002.

Krasnopolsky, V. M., Fox-Rabinovitz, M. S., and Chalikov, D. V.: New Approach to Calculation of Atmospheric Model Physics: Accurate and Fast Neural Network Emulation of Longwave Radiation in a Climate Model, Mon. Weather Rev., 133, 1370-1383, https://doi.org/10.1175/MWR2923.1, 2005.

Krasnopolsky, V. M., Fox-Rabinovitz, M. S., and Belochitski, A. A.: Using Ensemble of Neural Networks to Learn Stochastic Convection Parameterizations for Climate and Numerical Weather Prediction Models from Data Simulated by a Cloud Resolving Model, Advances in Artificial Neural Systems, 2013, 485913, https://doi.org/10.1155/2013/485913, 2013.

Le, Q. V.: Building high-level features using large scale unsupervised learning, in: 2013 IEEE International Conference on Acoustics, Speech and Signal Processing, 8595-8598, https://doi.org/10.1109/ICASSP.2013.6639343, 2013.
Lorenz, E. N.: Predictability - a problem partly solved, in: Predictability of Weather and Climate, edited by: Palmer, T. N. and Hagedorn, R., chap. 3, Cambridge University Press, 40-58, 2006.

Nooteboom, P. D., Feng, Q. Y., López, C., Hernández-García, E., and Dijkstra, H. A.: Using network theory and machine learning to predict El Niño, Earth Syst. Dynam., 9, 969-983, https://doi.org/10.5194/esd-9-969-2018, 2018.

O'Gorman, P. A. and Dwyer, J. G.: Using machine learning to parameterize moist convection: potential for modeling of climate, climate change and extreme events, ArXiv e-prints, arXiv:1806.11037, 2018.

Rasp, S. and Lerch, S.: Neural networks for post-processing ensemble weather forecasts, ArXiv e-prints, arXiv:1805.09091, 2018.

Rasp, S., Pritchard, M. S., and Gentine, P.: Deep learning to represent sub-grid processes in climate models, ArXiv e-prints, arXiv:1806.04731, 2018.

Schneider, T., Lan, S., Stuart, A., and Teixeira, J.: Earth System Modeling 2.0: A Blueprint for Models That Learn From Observations and Targeted High-Resolution Simulations, Geophys. Res. Lett., 44, 12396-12417, https://doi.org/10.1002/2017GL076101, 2018.

TensorFlow: available at: https://www.tensorflow.org, last access: 28 September 2018

Thornes, T., Düben, P., and Palmer, T.: On the use of scaledependent precision in Earth System modelling, Q. J. Roy. Meteor. Soc., 143, 897-908, https://doi.org/10.1002/qj.2974, 2017.

Tolman, H. L., Krasnopolsky, V. M., and Chalikov, D. V.: Neural network approximations for nonlinear interactions in wind wave spectra: direct mapping for wind seas in deep water, Ocean Model., 8, 253-278, 2005. 